\title{
REPRESENTACIONES SOCIALES RELACIONADAS A LA ANEMIA EN NIÑOS MENORES DE TRES AÑOS EN COMUNIDADES AWAJÚN Y WAMPIS, PERÚ
}

\author{
Julio Mayca-Pérez ${ }^{1,2, b, d}$, Armando Medina-Ibañez ${ }^{1, a, c}$, José E. Velásquez-Hurtado ${ }^{1,2, b, d}$, \\ Luis F. Llanos-Zavalaga ${ }^{1,2, b, e}$
}

\begin{abstract}
RESUMEN
Objetivos. Conocer las representaciones sociales de las comunidades awajún y wampis en torno a síntomas y tratamiento de la anemia en niños menores de 03 años, la relación con sus simbolismos, constructos y tipo de alimentación. Materiales y métodos. Estudio cualitativo realizado entre junio y agosto del 2015, en los distritos de Río Santiago, Cenepa y Nieva (región Amazonas, Perú); que incluyó entrevistas en profundidad (EP) para personal de salud, autoridades de la comunidad y padres de familia, y grupos focales (GF) para madres de familia. Resultados. Se realizaron 38 EP y 13 GF. La población no tuvo un significado claro sobre la anemia, ni manejaba la misma descripción del personal de salud; sino que lo relacionaba con una sintomatología en base a sus representaciones sociales y manifestaciones culturales. Esto aplicaba en la decisión de los padres por el tratamiento a optar, no necesariamente, lo indicado por el personal de salud, y que prefieren el consumo de ciertos alimentos que podrían curar el putsumat. El acudir al establecimiento de salud o utilizar multimicronutrientes no era entonces su primera opción de tratamiento. Conclusiones. Las representaciones sociales y prácticas tradicionales continúan existiendo y poseen sistemas interpretativos sobre la salud, la enfermedad y su manejo, con lógicas, sentidos y coherencia según grupo cultural. El putsumat o putsuju es un modelo interpretativo equivalente para la anemia, con una sintomatología presentada por los niños con palidez, delgadez, cansancio, sustentado en el sistema cultural simbólico de las poblaciones awajún y wampis.

Palabras clave: Investigación cualitativa; Percepción social; Grupos de población; Población indígena; Anemia, niño (fuente: DeCS BIREME).
\end{abstract}

\section{SOCIAL REPRESENTATIONS RELATED TO ANEMIA IN CHILDREN UNDER THREE YEARS IN AWAJÚN AND WAMPIS COMMUNITIES OF PERU}

\begin{abstract}
Objectives. To understand the social representations of the Awajún and Wampis communities related to the symptoms and treatment of anemia in children younger than 3 years, as well as the relationship of these representations with the symbolism, constructs, and type of diet of these populations. Materials and methods. This qualitative study was conducted from June to August 2015 in the districts of Río Santiago, Cenepa, and Nieva (Amazon region, Peru), and included in-depth interviews (IDIs) of health personnel, community authorities, parents, and focus groups (FGs) for mothers. Results. A total of 38 IDIs and $13 \mathrm{FGs}$ were conducted. The evaluated populations had limited awareness about anemia and health personnel, but anemia with symptoms were correlated with social representations and cultural manifestations. This behavior was reflected in the parents' choice of treatments that were not necessarily the same as those indicated by the health personnel, and these social groups preferred the consumption of certain foods that were considered to cure the "putsumat". Visiting a health care center or using micronutrients was not their first treatment option. Conclusions. Social representations and traditional practices still exist, and include interpretative systems in health, disease, and disease management. The logic, meaning, and coherence of these practices depend on the cultural group considered. The "putsumat" or "putsuju" is an interpretive model for anemia, and the symptoms in children include pallor, thinness, and fatigue; this model is based on the cultural system of the Awajún and Wampis populations.
\end{abstract}

Key words: Qualitative research; Social perception; Population groups; Indigenous population; Anemia, Child (source: MeSH NLM).

\section{Ministerio de Desarrollo Social e Inclusión Social. Lima, Perú.}

Universidad Peruana Cayetano Heredia. Lima, Perú.

Antropólogo, ${ }^{\mathrm{b}}$ médico, ${ }^{\mathrm{c}}$ doctor en Humanidades, ${ }^{\mathrm{d}}$ magíster en Salud Pública, ${ }^{\mathrm{e}}$ magíster en Políticas, Planificación y Financiamiento en Salud.

Recibido: 09/05/2017 Aprobado: 06/09/2017 En línea: 29/09/2017

Citar como: Mayca-Pérez J, Medina-Ibañez A, Velásquez-Hurtado JE, Llanos-Zavalaga LF. Representaciones sociales relacionadas a la anemia en niños menores de tres años en comunidades Awajun y Wampis, Perú. Rev Peru Med Exp Salud Publica. 2017;34(3):414-22. doi: 10.17843/rpmesp.2017.343.2870 


\section{INTRODUCCIÓN}

En el Perú, la desnutrición crónica infantil y la anemia son problemas de salud pública condicionados por determinantes sociales, culturales, económicos, climáticos, medioambientales y de desarrollo, asociados con la pobreza y brechas de inequidad ${ }^{(1)}$. La presencia de estos daños durante el crecimiento y la etapa de diferenciación cerebral generan consecuencias adversas principalmente en el desarrollo (2).

En Perú (2016), la prevalencia de anemia en menores de tres años, en el área rural fue del $56,0 \%$, de $51,7 \%$ en la selva y de $44,6 \%$ en la región Amazonas ${ }^{(3)}$. Un estudio desarrollado en la provincia de Condorcanqui - Amazonas mostró que la prevalencia de anemia en poblaciones indígenas en el 2012 fue de 51,3\%; esta población se caracteriza por la escasez de recursos económicos en el ámbito familiar, desconocimiento del valor nutricional de los alimentos, escasa participación ciudadana y social, y la brecha cultural que limitan el acceso a una buena nutrición de sus niños ${ }^{(5)}$.

En comunidades awajún, el cuidado y recuperación de la salud se relacionan con rituales o pedidos a la tierra (nugkui), al agua (tsugki) o plantas como el toé (6) Dichas prácticas se alejan de la medicina occidental, debido a que esta comunidad brinda a las enfermedades una explicación dentro de su lógica simbólica, asociándolas a la brujería, el "cutipado" u otras representaciones ${ }^{(7)}$.

El escenario descrito genera la necesidad de estudios cualitativos que profundicen en aspectos sociales, realidades de la vida y contextos culturales, a través de procedimientos rigurosos, sistemáticos y críticos ${ }^{(8)}$.

El objetivo del presente estudio es conocer las representaciones sociales de las comunidades awajún y wampis en torno a los síntomas y el tratamiento de la anemia en niños menores de tres años, la relación con sus creencias, costumbres y tipo de alimentación entre otros aspectos. Los resultados aportarán con el entendimiento y adecuación cultural a favor de la aceptabilidad del multimicronutriente (MMN).

\section{MATERIAL Y MÉTODOS}

Estudio cualitativo exploratorio y descriptivo realizado entre junio y agosto de 2015 en la provincia de Condorcanqui (distritos de Nieva, Río Santiago, y Cenepa) (Figura 1). Su población pertenece a los grupos étnicos awajún (cuencas de los ríos Cenepa, Santiago y Nieva), y los wampis (márgenes del río Santiago). Dichos grupos tienen características culturales similares por pertenecer a la misma familia lingüística. Si bien hay variaciones en pronunciación o escritura, la esencia del

\section{MENSAJES CLAVE}

Motivación para realizar el estudio. Estudios de la percepción y conocimientos del poblador nativo amazónico sobre la anemia y su tratamiento son escasos, siendo este un problema prioritario en la salud pública del país. La cultura amazónica mantiene representaciones paralelas al conocimiento occidental de la anemia.

Principales hallazgos. Los pobladores awajún y wampis consideran que el putsumat o putsuju es una enfermedad con síntomas similares a la anemia, su primera opción de tratamiento está en la medicina tradicional (por ejemplo, usando plantas); esto es paralelo a la medicina occidental que indica el uso de multimicronutrientes o sulfato ferroso.

Implicancias. Es necesario promover el conocimiento de la cultura indígena para generar estrategias que acerquen más el tratamiento contra la anemia a la comunidad.

significado se mantiene. Estos aspectos de similitud ya han sido observados en estudios anteriores $(5,11,16)$.

Para elegir las comunidades de estudio se consideraron que estas pertenezcan a los quintiles 1 y 2 de pobreza, que tengan mayor concentración poblacional y mayor

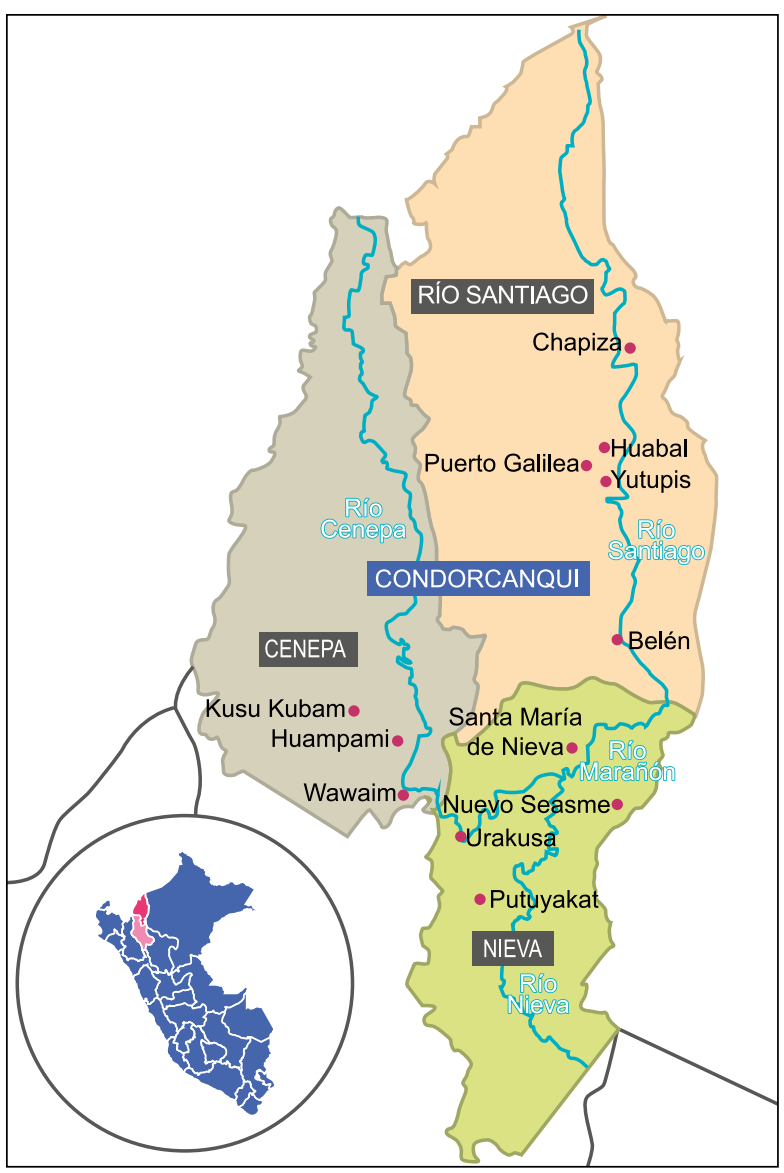

Figura 1. Comunidades awajún y wampis incluidas en el estudio sobre representaciones sociales relacionadas a la anemia, Perú 2015. 
acceso geográfico, que cuenten con niños entre 6 meses y 36 meses, y que cuenten con establecimiento de salud y profesional encargado de los MMN (además de lotes del producto), que realicen actividades de promoción y difusión de la suplementación.

Se utilizaron dos técnicas para recolectar información: 13 grupos focales (GF) y 38 entrevistas en profundidad (EP) (Tabla 1). Las EP estuvieron dirigidas a personal de salud (PS), agentes comunitarios (AgC), padres (padre o madre biológica) y autoridades de la comunidad (AC); y los GF a madres o cuidadores principales (CP). Las dimensiones y subdimensiones estudiadas fueron: sintomatología de la enfermedad, su tratamiento, relación del PS con la comunidad, alimentos relacionados, y uso de los MMN (Tabla 2). En los resultados se incluye, de manera concisa, los mensajes con mayor valor en la descripción de estas dimensiones.

Los instrumentos fueron validados previamente en un piloto de campo. Para la recolección de la información, se tuvo apoyo de traductores bilingües de la comunidad, quienes conocieron la investigación y el sentido de las preguntas para adecuar los términos al momento de la traducción. Se utilizó el software NVivo 11 (QSR international Pty Ltd, Australia) para codificar y sistematizar la información, que se agrupó y clasificó en temas y subtemas, y según tipo de actor, a partir de los formatos semiestructurado de los instrumentos, generando las categorías más frecuentes presentadas, y que permitió la triangulación de resultados.

El protocolo del estudio fue aprobado por el Comité Institucional de Ética de la Universidad Peruana Cayetano
Heredia, y se obtuvo el consentimiento informado verbal de cada participante, y permiso para las grabaciones, garantizándose la confidencialidad de la información.

\section{RESULTADOS}

\section{LA ENFERMEDAD Y SU SINTOMATOLOGÍA}

Según lo reportado por el PS que atiende a niños menos de tres años, la anemia no tiene una definición clara para la comunidad. El putsumat guarda estrecha relación con la anemia y se refleja en los síntomas: "flaco" (watsaju), "debilidad en la sangre", "baja energía", "cansancio", entre otros, y cuando se dan estas características en sus niños significa que algo anda mal.

...Queda débil, muy blanco, ese se llama putsumat que le dicen... Misma palabra dice qué es anemia, debilidad de sangre, baja energía y no tienes fuerza, tienes cansancio y no puedes hacer trabajos, te da sueño, no tienes habilidades, te desmoralizas y te sientes decaído (EP Autoridad Ebron).

Un niño enfermo con putsumat es identificado por la comunidad, además por su estado emocional como tristeza, desánimo para jugar, o características físicas como estar "barrigoncito" (que se podría relacionar con parasitosis) y retraso para comenzar a caminar. Dichas percepciones son bastante similares al preguntar sobre qué entienden por anemia, donde predomina la palidez y falta de alimentación.

Tabla 1. Comunidades awajún y wampis incluidas en el estudio sobre representaciones sociales sobre anemia, Perú 2015

\begin{tabular}{|c|c|c|c|c|c|c|}
\hline \multirow{2}{*}{\multicolumn{3}{|c|}{ Ámbito }} & \multicolumn{4}{|c|}{ Instrumento aplicado } \\
\hline & & & \multicolumn{2}{|c|}{ Entrevista en profundidad } & \multicolumn{2}{|r|}{ Grupo focal } \\
\hline Etnia & Distrito & Comunidad & $\begin{array}{c}\text { Líder y/o agente } \\
\text { comunitario }\end{array}$ & $\begin{array}{c}\text { Padre o madre de } \\
\text { familia }\end{array}$ & $\begin{array}{l}\text { Personal } \\
\text { de salud }\end{array}$ & $\begin{array}{c}\text { Madre de } \\
\text { familia }\end{array}$ \\
\hline \multirow[t]{2}{*}{ Awajun } & Río Santiago & Belén & 1 & 1 & 1 & 1 \\
\hline & & Yutupis & 1 & 1 & 1 & 1 \\
\hline \multirow[t]{3}{*}{ Awajun } & Cenepa & Huamapami & 1 & 1 & 1 & 1 \\
\hline & & KusuKubaim & 1 & 1 & 1 & 1 \\
\hline & & Wawain & 1 & 1 & 1 & 1 \\
\hline \multirow[t]{3}{*}{ Awajun } & Sta. María de Nieva & Nieva & 1 & 1 & 1 & 1 \\
\hline & & Urakusa & 1 & 1 & 1 & 1 \\
\hline & & $\begin{array}{l}\text { Nuevo } \\
\text { Seasme }\end{array}$ & 1 & 1 & 1 & 1 \\
\hline \multirow[t]{4}{*}{ Wampis } & Río Santiago & Puerto Galilea & 1 & 1 & 1 & 1 \\
\hline & & Chapiza & 1 & 1 & 1 & 1 \\
\hline & & Guayabal & 1 & 1 & 1 & 1 \\
\hline & & Huabal & 1 & 1 & 1 & 1 \\
\hline Total & & & 13 & 12 & 13 & 13 \\
\hline
\end{tabular}


Tabla 2. Dimensiones del estudio desarrollado en comunidades awajún y wampis sobre representaciones sociales relacionadas a la anemia, Perú 2015

\begin{tabular}{|c|c|c|}
\hline Dimensión & Definición operacional & Subdimensiones \\
\hline $\begin{array}{l}\text { La enfermedad y su } \\
\text { sintomatología }\end{array}$ & $\begin{array}{l}\text { Conjunto de representaciones simbólicas y } \\
\text { culturales que las personas construyen en torno a la } \\
\text { anemia y al putsumat, basados en su idiosincrasia y } \\
\text { sistemas culturales. }\end{array}$ & $\begin{array}{l}\text { - Representación de la enfermedad. } \\
\text { - Concepción simbólica de los alimentos } \\
\text { - Información biomédica sobre la anemia } \\
\text { frente a la concepción sociocultural de la } \\
\text { misma. }\end{array}$ \\
\hline $\begin{array}{l}\text { Tratamiento de la } \\
\text { enfermedad }\end{array}$ & $\begin{array}{l}\text { Es la manera de como los usuarios de los } \\
\text { establecimientos de salud comprenden e interpreta } \\
\text { el tratamiento que se les ofrece para la anemia o el } \\
\text { putsumat. }\end{array}$ & $\begin{array}{l}\text { - Significación sociocultural del tratamiento } \\
\text { - Tratamientos biomédicos de la anemia y } \\
\text { concepción cultural de la misma. }\end{array}$ \\
\hline $\begin{array}{l}\text { Relación entre el personal } \\
\text { de salud y la comunidad }\end{array}$ & $\begin{array}{l}\text { Es la manera de como el personal de salud observa } \\
\text { e interpreta el modo de vida, costumbres, idioma, } \\
\text { cultura y como se relacionan los individuos de una } \\
\text { determinada comunidad. }\end{array}$ & $\begin{array}{l}\text { - Conocimiento de la cultura del otro. } \\
\text { - Percepción de su función laboral en la } \\
\text { comunidad. } \\
\text { - Satisfacción laboral y con su entorno. } \\
\text { - Empatía sociocultural. }\end{array}$ \\
\hline $\begin{array}{l}\text { Alimentos relacionados con } \\
\text { la enfermedad }\end{array}$ & $\begin{array}{l}\text { Ingesta de alimentos para proveer de los nutrientes } \\
\text { necesarios para el cuerpo y su desarrollo. Incluye } \\
\text { alimentos relacionados al tratamiento del putsumat. }\end{array}$ & $\begin{array}{l}\text { - Alimentos para consumo de los niños. } \\
\text { - Momentos de alimentación. } \\
\text { - Productos disponibles en la naturaleza. }\end{array}$ \\
\hline $\begin{array}{l}\text { Uso de los MMN como } \\
\text { tratamiento }\end{array}$ & $\begin{array}{l}\text { Uso adecuado del MMN que incluye la preparación } \\
\text { del mismo disolviéndolo en alguna comida, además } \\
\text { que sea ingerido el total de la dosis. }\end{array}$ & $\begin{array}{l}\text { - Capacitación en uso de los MMN. } \\
\text { - Confianza y aceptabilidad al MMN. }\end{array}$ \\
\hline
\end{tabular}

MMN: multimicronutrientes.

Esta enfermito. ¿Qué puede tener? Anemia, bichos... A veces cuando no cuidamos bien, cuando no le damos comida bien, de eso mayormente, de parásitos... Se pone posheco (delgado, pálido, desganado), a veces hincha su carita, se pone triste, no está bien, se pone a su cama a dormir, eso también (GF Madres Guayabal).

El putsumat (Nieva) o putsuju (Río Santiago), es referido con características propias no tan concretas como cuando es descrita la anemia por un PS. Las madres son las únicas que pueden dar el tratamiento a sus hijos, lo que requiere el uso de plantas medicinales y dietas que ellas conocen y describen y, como dice una madre: Solo si no se sana lo llevaré al centro de salud ya que el uso de las plantas medicinales es usual antes de acudir con un PS, quienes poco a poco van entendiendo estos aspectos culturales.

...Esa confusión debe haber porque putsuju es muy diferente de la anemia, entonces... Sí yo he visto, el putsujú cambia de color se vuelve blanco, pálido. El pelo cambia también está medio amarillento. Algunos hinchaditos la cara. Eso es putsuju; también tiene diarrea, sueño, bajo apetito (EP AgC Yutupis).

\section{TRATAMIENTO DE LA ENFERMEDAD}

Hay diferencias entre el tratamiento biomédico y el tradicional. Para el primero, lo usual es seguir las recomendaciones del PS para tratar la anemia, lo cual inicia con la evaluación del niño en la consulta de crecimiento y desarrollo (CRED), dosaje de hemoglobina, brindar tratamiento para desparasitación y luego los MMN (o sulfato ferroso) como tratamiento específico de la anemia, además de brindarles sesiones demostrativas para el adecuado preparado de alimentos.

Para que las mamás vean... acepten. Yo primero hago tamizaje de hemoglobina... A partir de 6 meses la anemia le va agarrar, ... Solamente quieren leche, lactancia materna, teta no más quieren y no comen y le va a producir anemia. Por eso, para que no produzca anemia tienes que dar chispitas. Yo le digo así a la mamá... tu hija tiene anemia, es una enfermedad, para curar tienes que dar vitamina... (EP AgC Huabal).

El putsumat refleja la idiosincrasia de la comunidad, explica que las causas están relacionadas a efectos espirituales o simbólicos, y puede llevar a la muerte si no se trata adecuadamente. La madre brinda el tratamiento dentro de un contexto ritualizado que incluye la obtención de la planta medicinal y la preparación de la infusión. El putsumat se trata con plantas medicinales como el tuyuc o el unkush, que sirven para dar fuerza y energía, y recuperar la sangre.

Tenemos una planta como vitamina, en mi idioma se llama: unkush, recupera la sangre y la fuerza, te da energía. Se prepara con la hoja verde y se chapea y se le da cuando el adulto está enfermo, con anemia, y se recupera, le damos tres veces, en distintos días o en un día seguido, no hace daño es planta original. En el caso de los niños le damos cocinadito, con sopita, metiendo pescadito y se recupera (EP Padre KusuKubain). 
El tuyuc tiene un color particular, rojo intenso. Se asa al fuego por unos segundos y se extrae un jugo rojo, que el niño tomará tres cucharaditas diariamente (Figura 2). Las madres mencionaron además que el MMN no podía hacer bien al niño pues era de color blanco y no rojo como la sangre. Ver a modo de resumen la Tabla 3.

\section{RELACIÓN DEL PERSONAL DE SALUD CON LA COMUNIDAD}

EI PS se involucra con la comunidad considerando su cultura, costumbres y formas de vivir, aunque en su mayoría son foráneos y de realidades distintas a la de los pueblos awajún y wampis. Entienden la importancia de este involucramiento para lograr un mayor grado de empatía con la población y darles mensajes más apropiados y entendibles. Un enfoque intercultural les permitirá posicionarse progresivamente en la mente de los usuarios, brindando un mejor servicio.

...Claro que sí (es importante la interculturalidad) porque te hacen conocer más la cultura... tienes dos culturas acá... Los mestizos y los awajún y como que ahí te hacen ver más la realidad de dónde estás. La cultura

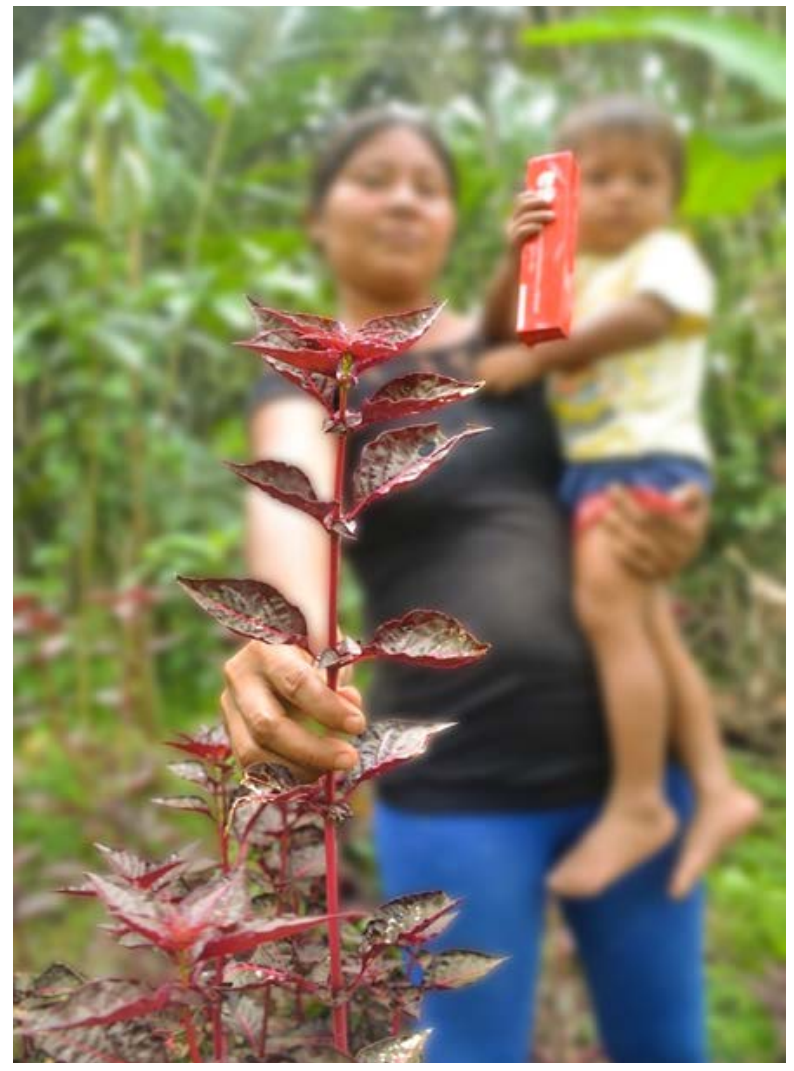

Fotografiado por: Armando Medina

Figura 2. Madre awajún muestra la planta tuyuc usada para curar el putsumat, mientras su niño muestra una caja de MMN aún sin consumir. awajún, que tú vienes con una de mestizo y te quedas ahí no más y no investigas más, no lees o no te interesa como se dice y con la interculturalidad ves más (EP PS Nuevo Seasme).

El manejo del idioma es fundamental, entendiendo que estas poblaciones hablan awajún, wampis y algunos un limitado castellano (menor en las mujeres). Estas poblaciones, en su mayoría, se comunican a través de la oralidad (mitos, cuentos, leyendas). El aproximarse y generar confianza con ellos a través del manejo del mismo idioma y la interculturalidad facilitaría las atenciones, además permitiría brindar un buen trato.

...Hay bastante persona de acá, ... nativas que manejan diferente idioma... te entienden una, dos, tres palabras en español y lo resto no, cuando tú quieres explicarle o explayarte más ellos ya no te entienden. Tengo que tener prácticamente alguien, digamos un apoyo, para que me lo traduzca y me los explique en su propio idioma (EP PS Galilea).

\section{ALIMENTOS RELACIONADOS CONLAENFERMEDAD}

Se hace una precisión en torno a un niño enfermo o watsaju (contextura delgada, flaco), que no acepta comidas. La madre insiste al niño con su alimentación, pero esto no es una práctica recurrente ya que, normalmente, el niño recibe los alimentos y lo dejan comiendo solo, pues las madres salen de casa a realizar labores agrícolas y no siempre llevan a sus hijos.

... Yo le doy temprano, empiezan a comer maduro, ... y cuando hay papaya me piden papaya, ... Si tengo platita tengo mi arrocito compradito, hago sopita, a veces hago un arroz sequito, si hay guisito hago sequito de carnecita o con huevito frito le hago almorzar... Cuando vengo de mi chacra junto verduritas que son del montecito, llego, le cocino y les doy. Pero eso casi no les gusta, verduras no comen. Me dicen: "amargo es mamá" y lo dejan. Pero cuando tengo platita le compro repollo, si le comen, ensaladita de repollo si comen, ensaladita de cebolla (EP cuidadora Guayabal).

Estas representaciones guardan relación con el consumo de alimentos y la variedad de productos obtenidos en su mayoría de la recolección, que solo se encuentran de acuerdo a las estaciones y ciclos biológicos de la naturaleza; por ejemplo, el "mijano", periodo en el que los peces aumentan en los ríos y quebradas debido al decrecimiento de las aguas. También se indican las diferencias entre la alimentación del pasado, variada en animales silvestres, y la actual.

... Antes había más alimentación, mis paisanos cazadores iban al monte a traer animales, carnes 
frescas, chontas de frontera y ya alimentaban sus niños... del bosque se trae chonta, suri, algún pájaro, perdiz, pescado, algún pescado chiquito, de una quebradita viene echa un barbasco, lo matan, traen con pijuayo bien preparado, ese es lo que invitaba a sus niños y lo alimentaba. Pero ahora ya no... difícil encontrar animales... pescado se come en época de mijano empieza agosto, setiembre, octubre. Tres meses. Hay harto pescad (EP AC KusuKubain).

Tal como se refiere, antes contaban con abundantes alimentos pues en el monte y el río se hallaban variedad y cantidad de animales, verduras y frutas. Hoy, esos productos escasean significativamente y las familias ya no tienen qué llevar a sus casas, pues lo que se cultiva en las chacras se rige por temporadas, tiempo de maduración y ciclos biológicos.

... Antes nuestros padres estaban bien alimentados, bien nutridos porque comian todo tipo de verduras del bosque. Cuando iban al monte traían cantidad de animales, pescaban en todas las quebradas, aves de monte... de repente la mujer se embarazaba, apenas cuando tenía antojo su marido empezaba a buscar... se iba al monte y traía pava, paujiles, chonta, suri, verduras, todo lo que la señora quería comer. Cuando ya daba a luz, también se iba, para que la señora no tenga anemia... Cuando no daba leche, se iba a sacar chonta y se curaba también,... Con la raíz de pijuayo, calentando también se sobaba para que dé más leche... Cuando su niño empezaba a comer, iba al monte, traía chonta y le cocinaba. Esa chonta purito le daba de comer de 6 meses. Alimentaba...más que le daba de comer al niño y más fuerte e inteligente salía (EP AC Wawain).

\section{USO DE LOS MMN COMO TRATAMIENTO}

EI PS desarrolla en base a su aprendizaje y experiencia, su concepción sobre los MMN; sin embargo, la composición del mismo y la aparición de síntomas no deseados o eventos adversos todavía no están claros algunos de ellos. Además, la mayoría solo recibió una capacitación y no necesariamente en base a la última normativa del Ministerio de Salud.

... Para mí la suplementación con MMN, las chispitas, es muy complicado, la madre no se niega a llevarlo, te recibe... parecen que te recibieran con mucho gusto, pero si tú vas a sus casas encuentras a algunas que lo tienen en bolsas guardadas, cuatro o cinco cajas ¿Por qué no le has dado? Y me dicen... me olvidé o les hace daño... las chispitas lo mezclan con dos gotas de alcohol y forman goma... o, lo utilizan como veneno para hormigas... Entonces, a ellos eso les impacta... (EP PS KusuKubain).

En relación a la percepción de los MMN de la comunidad, se deben considerar dos temas, la confianza, que se expresa en la aceptabilidad y el deseo de conocer más sobre los MMN y sus componentes; y la desconfianza, que se expresa de diferentes maneras, para ello la población toma sus propias precauciones a fin de corroborar lo indicado en los establecimientos de salud, con el fin de verificar los efectos de un producto desconocido.

Tabla 3. Relación entre anemia y putsumat o putsuju según las representaciones sociales relacionadas a la anemia en comunidades awajún y wampis, Perú 2015.

\begin{tabular}{|c|c|c|c|c|}
\hline Visión & Actor & Signos y síntomas & Tratamiento & Prevención \\
\hline $\begin{array}{l}\text { Occidental: } \\
\text { anemia }\end{array}$ & $\begin{array}{l}\text { Prestador de } \\
\text { salud, agente } \\
\text { comunitario }\end{array}$ & $\begin{array}{l}\text { - Debilidad, cansancio, sueño } \\
\text { - Se hace flaquito } \\
\text { - No camina } \\
\text { - Tiene diarrea } \\
\text { - No tiene apetito } \\
\text { - Tiene mareos, dolor de cabeza, mira borroso } \\
\text { - Para enfermo } \\
\text { - Carita blanca } \\
\text { - La sangre no le funciona normal }\end{array}$ & $\begin{array}{l}\text { Sulfato ferroso } \\
\text { MMN } \\
\text { Antiparasitarios }\end{array}$ & $\begin{array}{l}\text { - Lactancia materna exclusiva } \\
\text { - Leche artificial } \\
\text { - Carne (res, chancho) } \\
\text { - Hígado (res, pollo) } \\
\text { - Pescado } \\
\text { - Menestras }\end{array}$ \\
\hline $\begin{array}{l}\text { Indígena: } \\
\text { putsumat } \\
\text { putsuju }\end{array}$ & $\begin{array}{l}\text { Comunidad, } \\
\text { cuidadores }\end{array}$ & $\begin{array}{l}\text { - Cambia de color, se vuelve blanco, pálido } \\
\text { - Pelo amarillento } \\
\text { - Hinchazón de la cara } \\
\text { - Diarrea } \\
\text { - Sueño, cansancio } \\
\text { - Bajo apetito } \\
\text { - Flaco } \\
\text { - Debilita la sangre } \\
\text { - Baja la energía, no tiene fuerza } \\
\text { - No tienes habilidades } \\
\text { - Desmotivación y decaimiento emocional }\end{array}$ & Tuyuc & $\begin{array}{l}\text { - Pescado (boquichico) } \\
\text { - Unkush (alimento nativo) } \\
\text { - Carne de monte (majaz, } \\
\text { paujil, sachavaca) }\end{array}$ \\
\hline
\end{tabular}


...Primera vez que han recibido estas chispitas (MMN), ... y los padres a veces decimos: ¿Por qué en la ciudad no se recibe eso? ...He visto un caso que un niño ha comido chispita de acuerdo a la indicación que ha dado la enfermera, comió chispita y se hincho todo su cuerpo, y como el pueblo es pequeño y una vez que ve ese tipo de reacción, propaga, y dice: eso está mal, eso es para engordar perros, nos están dando para morir. Viendo esos casos, ... ya no quieren recibir... a veces lo botan (EP Padre KusuKubain).

La comunidad necesita conocer en mayor profundidad los MMN para poder otorgarles más confianza y aceptabilidad, y esto depende de la sensibilización recibida, la cual no solamente debe ser verbal, pues es más directo de modo visual, incluso a través de resultados comparativos entre niños que consumen y otros que no, para ver (de algún modo) el grado de evolución, es decir cómo se encontraba el niño inicialmente y luego de un periodo de consumir MMN. Los conceptos para la preparación y consumo del MMN deben reforzarse en cada consulta para que la madre confíe más en el producto.

...Mi hijito no come cuando le mezclo todo en dos cucharas, no come. Yo le estoy dando así, saco 2 cucharas, pongo media nada más de chispitas, porque si le hecho todo hay otro sabor... Lo vomita, cuando le doy todo mezclado, por eso estoy dando medio sobrecito y ahí come, cuando termina y pide todo lo que hay... Yo cuando le toca control a mi hijito, quiero que me expliquen, pero no hacen asi". (GF Madres Puerto Galilea)

Sobre los MMN observamos diferentes connotaciones sustentadas en el simbolismo colectivo, por ejemplo, se asocia a los MMN con imágenes diabólicas y, por ende, al mal. De allí que se observa resistencia a su consumo para no sufrir efectos nocivos. Otras madres reciben el producto pero no lo consumen porque no existe una costumbre que ayude al consumo por periodos prolongados.

\section{DISCUSIÓN}

Se plantean dos modelos interpretativos y explicativos relacionados con la anemia. El modelo biomédico explica la anemia a partir de la deficiencia de hierro principalmente $y$, por otro lado, las representaciones de las poblaciones estudiadas, sustentadas en un sistema cultural simbólico, que identifican algunas características de esta enfermedad, a partir de los discursos de los actores locales, y sustentadas en su idiosincrasia y desarrollo histórico social ${ }^{(9,10)}$.

La medicina occidental explica la anemia como producto del déficit de micronutrientes como el hierro, y se manifiesta a través de síntomas como palidez, cansancio, etc. La cosmovisión amazónica, sin embargo, entiende a la anemia como una enfermedad que ha llegado a estas poblaciones en tiempos no muy lejano. Con el discurso del PS, y dentro de su lógica cultural, a la sintomatología presentada por los niños con palidez, delgadez, cansancio, etc., le denomina putsumat o putsuju. Por motivos explicativos, y en base a las dimensiones propuestas para este estudio, podemos comparar ambos síndromes y sus síntomas, sin que uno sea el equivalente al otro, cada cual con su propio diagnóstico y tratamiento (Tabla 3).

Para las comunidades nativas, las enfermedades se comprenden en función a síndromes culturales, mágicos o religiosos, como por ejemplo el cutipado, mal de ojo, o el susto, que se asocian a alguna energía negativa que se ha adquirido de algún animal o lugar, esto incluso se trasmite de la madre al niño desde el embarazo ${ }^{(11,12)}$. Estos síndromes según la psiquiatría transcultural, constituyen patrones recurrentes de comportamiento aberrante o experiencias problematizadoras relacionadas con un ámbito geográfico o con algún diagnóstico médico ${ }^{(12,13)}$.

El desarrollo de formas y saberes diferenciados o antagónicos al biomédico, se relaciona con las condiciones religiosas, étnicas, económicas, políticas, técnicas y científicas, que generan representaciones sociales que circunscriben estas formas de padecimientos o síndromes culturales, relacionados a complejos sistemas de sintomatologías provocados por transgredir ciertas normas sociales, espirituales o ambientales ${ }^{\left({ }^{10}\right)}$. El rompimiento de ciertos tabú o simbolismos mágicos que circunscriben a los alimentos o el desacato de estas normas, lleva a los individuos a la enfermedad, como por ejemplo el cutipado ${ }^{(11,14)}$.

La población al identificar una enfermedad o padecimiento, aprovecha los recursos vegetales, animales o minerales disponibles en su entorno, así, por ejemplo, usa plantas medicinales o verduras del bosque que consumen como función terapéutica, pues asumen que comiendo determinados productos superarán su problema de salud (10,15-17). Un ejemplo observado es en los miskitu (Nicaragua), donde para la anemia o palidez usan la cuculmeca (Dusa pauni), que se hierve hasta que el agua se pone roja ${ }^{(18)}$.

Otro estudio en ocho países (Bolivia, Burkina Faso, Guatemala, Honduras, India, Indonesia, Malauí y Pakistán), verificó que las mujeres desconocían el término anemia, pero sí distinguían sus síntomas: dolor de cabeza, mareo, palidez y sangre "fina" o "débil". Su tratamiento incluía comida nutritiva, vitaminas, medicinas, y recursos caseros como hierbas y plantas. Creían que la ingestión de hierro "puede causar exceso de sangre o un bebé grande", manchas en el rostro del bebé u otro daño ${ }^{(19)}$. Comportamientos similares en relación a uso y confianza en plantas medicinales o prácticas ritualizadas, encontramos también a nivel nacional ${ }^{(11,16,20)}$. 
Las representaciones sociales y prácticas tradicionales fueron, durante mucho tiempo, consideradas exóticas, desprovistas de coherencia y eficacia, características de sociedades y culturas subdesarrolladas, destinadas a desaparecer por la implementación y diseminación de la medicina occidental ${ }^{(21)}$. A pesar de estos calificativos y predicciones, estas continúan existiendo, y hoy se sabe que poseen sistemas interpretativos, algunos más elaborados que otros, sobre la salud, la enfermedad y su manejo, con lógicas, sentidos y coherencia dentro del contexto sociocultural de cada grupo ${ }^{(22)}$.

Los factores socioeconómicos, geográficos y étnicos afectan el desarrollo y nutrición del niño, siendo necesario invertir en mejorar la educación, empleabilidad, vivienda y servicios básicos ${ }^{(23)}$, así como mayor sensibilización a la comunidad sobre la anemia. Es necesario escuchar a la población indígena y diseñar en forma conjunta estrategias congruentes con sus condiciones de vida ${ }^{(20,24)}$, en camino a acercar los servicios de salud donde encuentren una atención de calidad ${ }^{(25)}$. Si el sector Salud quiere conocer y/o implementar un sistema de atención más cercano a la comunidad, debe identificar, describir y analizar las formas y saberes que los sujetos y conjuntos sociales manejan sobre los padeceres reales o imaginarios que afectan su salud (10).

Para captar los matices y sutilezas de esta medicina doméstica es necesario indagar, mediante el ejercicio de la etnografía y estudios de caso concretos, en la dinámica existente entre las distintas opciones terapéuticas de un medio cultural específico y en el sistema cosmológico que sustenta la construcción simbólica de la enfermedad y los recursos para su tratamiento ${ }^{(26)}$.

Se sugiere capacitar y sensibilizar al PS en temas de: interculturalidad, nutrición, comunicación intercultural y educación de adultos, además de brindarles materiales educativos e informativos sobre estos temas, y que incorporen en su atención conocimientos sobre los recursos vegetales y animales, y las diversas costumbres existentes según ámbito geográfico.

Contribuciones de autoría: JMP, AMI, JEVH Y LLLZ participaron en la concepción y diseño del artículo. AMI participó en la recolección de los resultados. JMP y AMI participaron en el análisis e interpretación de datos, y en la redacción del artículo. JMP, AMI, JEVH Y LLLZ participaron en la aprobación final del artículo.

Fuentes de financiamiento: la información se recopiló a partir de la consultoría: "Percepciones y prácticas de las familias, actores locales y personal de salud respecto al consumo de multimicronutrientes en niñas y niños de 06 meses a menos de 36 meses de edad en la provincia de Condorcanqui-Amazonas", financiada por el Ministerio de Desarrollo e Inclusión Social.

Declaración de conflictos de interés: los autores declaran no tener conflictos de interés

\section{REFERENCIAS BIBLIOGRÁFICAS}

1. Lazarte CE, Soto A, Alvarez L, Bergenståhl B, Medrano N, Granfeldt Y. Nutritional status of children with intestinal parasites from a tropical area of Bolivia, emphasis on zinc and iron status. Food Nutr Sci. 2015; 6(4):399-411. doi: 10.4236/fns.2015.64041

2. Black MM, Walker SP, Fernald LCH, Andersen CT, DiGirolamo AM, Lu C, et al. Early childhood development coming of age: science through the life course. Lancet. 2017;389(10064): 77-90. doi: 10.1016/S0140-6736(16)31389-7

3. Instituto Nacional de Estadística e Informática (INEI). Perú: Encuesta Demográfica y de Salud Familiar 2016, Nacional y Regional [Internet]. Lima: INEI; 2017 [citado el 15 de mayo 2017]. Disponible en: https://www.inei.gob.pe/ media/MenuRecursivo/publicaciones digitales/Est/Lib1433/index.html

4. Organización Panamericana de la Salud (OPS). Informe técnico: Estado de salud y nutrición de los niños menores de 5 años pertenecientes a las poblaciones indígenas y no indígenas de Bagua y
Condorcanqui en la Región Amazonas 2012 [Internet]. Lima, Perú: OPS/ OMS; 2013 [citado el 15 de mayo 2017]. Disponible en: http://www.bvsde. paho.org/documentosdigitales/bvsde/ texcom/000020.pdf

5. Plan Binacional de Desarrollo de la Región fronteriza Perú-Ecuador. Desarrollo Humano: Desarrollo sostenible en el Río Santiago. Línea Base. Lima: UNICEF; 2003.

6. Chocano L. Hombres y mujeres del Alto Marañón: El pueblo Awajún y su percepción del Cambio Climático. Bolivia: SPC Impresores S.A.; 2010.

7. Torres F. Nugkui; inspiración de las mujeres Awajún en el manejo de la biodiversidad para su seguridad alimentaria en el alto Marañon. Lima: Ediciones SAIPE; 2013.

8. Mieles MD, Tonon G, Alvarado SV. Investigación cualitativa: el análisis temático para el tratamiento de la información desde el enfoque de la fenomenología social. Univ Humanist. 2012;74(2):195-225.
9. Belaunde L. El recuerdo de Luna: género, sangre y memoria entre los pueblos amazónicos. Lima, Perú: Fondo Editorial de Ciencias Sociales, UNMSM; 2005.

10. Menéndez E. De sujetos, saberes y estructuras: introducción al enfoque relacional en el estudio de la salud colectiva. Buenos Aires: Lugar Editorial; 2009.

11. Medina A, Mayca J. Creencias y costumbres relacionadas con el embarazo, parto y puerperio en comunidades nativas Awajún y Wampis. Rev Peru Med Exp Salud Publica 2006;23(1): 22-32.

12. Cáceres E. Tejiendo las bases teóricas del sistema medico indígena andino: salud e interculturalidad desde los andes. Cusco, Perú: Universidad Nacional San Antonio Abad del Cusco; 2015.

13. Lámbari A, Flores $F$, Berenzon $S$. Curanderos, malestar y "daños": una interpretación social. Salud Ment. 2012; 35(2): 123-8.

14. Achig DR. Análisis hermenéutico de los saberes andinos y salud en El Tambo, provincia del Cañar, 2016 [Tesis para optar el grado de Magister]. Cuenca, 
Ecuador: Universidad de Cuenca; 2017 [citado el 15 de mayo de 2017]. Disponible en: http://dspace.ucuenca. edu.ec/handle/123456789/27023

15. Chirif A. Salud y nutrición en sociedades nativas. Lima: Centro de Investigación y Promoción Amazónica; 1979.

16. Cáceres A. Costumbres y creencias relacionadas con la salud en las comunidades nativas awajún y wampis de la región Amazonas. Ciencias Sociales y Humanidades 2016; 1(1): 9-15

17. Mantilla BP, Oviedo MP, Hernández A, Flórez NZ. Educación para la salud: una experiencia con población indígena del departamento de Chocó. Hacia Promoc Salud. 2013;18(2):96-109.

18. Grádiz LC, Garth S. Creencias y costumbres tradicionales del pueblo indígena Mískitu de Kahkabila. Cienc Intercult. 2009;5(2):105-15.

19. Galloway R, Dusch E, Elder L, Achadi E, Grajeda R, Hurtado E, et al. Women's perceptions of iron deficiency and anemia prevention and control in eight developing countries. Social Science \& Medicine 2002; 55(4):529-44.

20. Del Pino P, Mena M, Torrejón S, Del Pino E, Aronés M, Portugal T. Repensar la desnutrición: infancia, alimentación y cultura en Ayacucho, Perú [Internet]. Lima, Perú: Instituto de Estudios Peruanos; 2012 [citado el 12 de abril 2017]. Disponible en: https:// www.accioncontraelhambre.org/sites/ default/files/documents/07._repensar_ la_desnutricion.pdf

21. Baia AC, Ferreira A, Sampaio DL, de Sena LX, Rodrigues V, de Azevedo VL. [Antropologia da saúde e da doença: contribuições para a construção de novas práticas em saúde]. Rev NUFEN. 2012;4(2),11-21. Portugués.

22. Alcaraz GM, Yagari L. La concepción de la curación chamánica entre los indígenas Embera de Colombia: un proceso de comunicación socio-cultural y fisiológico. Invest Educ Enferm. 2003;21(2):60-78.

23. Larrea C, Freire W. Social inequality and child malnutrition in four Andean countries. Rev Panam Salud Publica. 200211(5-6):356-64.

24. Huayhua M. Documento de trabajo 147, Discriminación y exclusión: políticas públicas de la salud y poblaciones indígenas. Lima: Instituto de Estudios Peruanos; 2006.

25. Montero E. Percepción de los habitantes indígenas de áreas rurales respecto al primer nivel de atención. El caso del sureste de Veracruz, México. Salud Colectiva 2011;7(1): 73-86.

26. Lorente $D$. Medicina indígena y males infantiles entre los Nahuas de Texcoco: pérdida de la guía, caída de mollera, tiricia y mal de ojo. An. Antrop. 2015;49(2):101-48. doi: 10.1016/ S0185-1225(15)30005-9

Correspondencia: Julio Mayca Pérez Dirección: Calle Juan de la Fuente 608 Miraflores. Lima, Perú.

Teléfono: (+51) 999336848

Correoelectrónico:juliomayca@gmail.com

\section{Suscríbete a nuestro canal de Youtuhte y disfruta la mejor y más completa información de investigación científica}

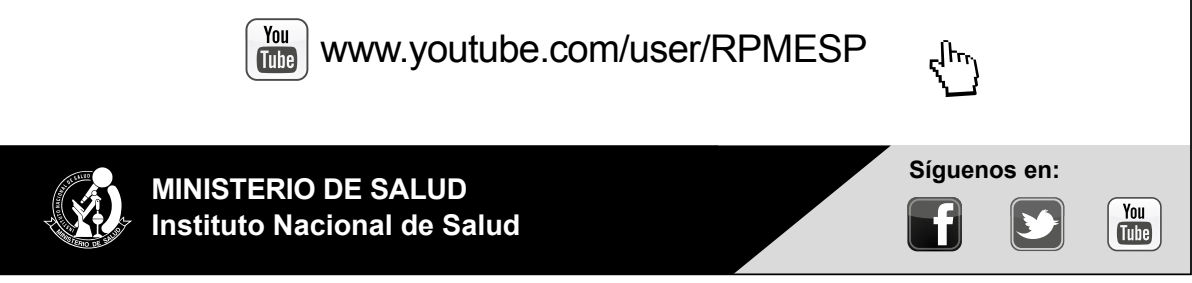

